03

\title{
Моделирование окисления расплава активной зоны ядерного реактора при наличии оксидной корки на поверхности расплава
}

\author{
() В.Б. Хабенский, ${ }^{1}$ В.И. Альмяшев,,$^{1,2}$ В.С. Грановский, ${ }^{1}$ Е.В. Крушинов, ${ }^{1}$ С.А. Витоль, ${ }^{1}$ \\ С.Ю. Котова, ${ }^{1}$ В.В. Гусаров ${ }^{3}$ \\ ${ }^{1}$ Научно-исследовательский технологический институт им. А.П. Александрова, \\ 188540 Сосновый Бор, Ленинградская обл., Россия \\ ${ }^{2}$ Санкт-Петербургский государственный электротехнический университет им. В.И. Ульянова (Ленина) „ЛЭТИ“, \\ 197376 Санкт-Петербург, Россия \\ ${ }^{3}$ Физико-технический институт им. А.Ф. Иофффе РАН, \\ 194021 Санкт-Петербург, Россия \\ e-mail: vac@mail.ru
}

Поступило в Редакцию 26 мая 2020 г.

В окончательной редакции 28 августа 2020 г.

Принято к публикации 1 сентября 2020 г.

\begin{abstract}
При тяжелой аварии АЭС с легководными реакторами наиболее эффективным способом локализации образующегося расплава (кориума) является его удержание в охлаждаемом корпусе реактора, сохранение целостности которого зависит от величины теплового потока от расплава к корпусу. При этом одним из критически важных процессов является окисление расплава водяным паром или паровоздушной смесью, которое может приводить к существенному увеличению тепловой нагрузки на корпус реактора за счет тепла экзотермических реакций окисления присутствующих в расплаве восстановителей, уменьшения толщины металлической составляющей ванны расплава, а также к выделению водорода, зависящих от скорости окисления. При рассмотрении условий окисления расплава учитывается, что для принятых сценариев тяжелой аварии наиболее реалистичной ситуацией является наличие твердофазного оксидного слоя (оксидной корки) на поверхности расплава. В указанных условиях на основе модели диффузии предложена зависимость для расчета скорости окисления расплава активной зоны ядерного реактора и выполнена ее валидация с использованием полученных экспериментальных данных.
\end{abstract}

Ключевые слова: тяжелая авария, расплав активной зоны, кориум, окисление, модель массопереноса, фокусирующий эффект.

DOI: 10.21883/JTF.2021.02.50356.181-20

\section{Введение}

Тяжелые аварии на АЭС, сопровождающиеся расплавлением активной зоны ядерного реактора с возможным последующим разрушением (разгерметизацией) корпуса реактора, несут в себе большие риски радиоактивного загрязнения окружающей среды $[1,2]$. По этой причине существует необходимость совершенствования имеющихся и создания новых систем безопасности АЭС, сводящих к возможному минимуму потенциальные риски таких аварий [3-5]. В настоящее время на основе анализа произошедших тяжелых аварий и опыта борьбы с их последствиями принят принцип глубокоэшелонированной защиты, опирающийся на множественность и дублирование уровней защиты и включающий последовательность барьеров на пути выхода радиоактивных материалов в окружающую среду [6]. При этом для обоснования работоспособности как существующих, так и разрабатываемых систем защиты проводились и проводятся экспериментальные исследования и теоретический анализ процессов, в том числе, а в последнее время преимущественно, физикохимических процессов, протекающих на всех стадиях тяжелой аварии [7-13].
С точки зрения простоты реализации, стоимостных характеристик и ликвидации последствий аварии из двух способов локализации расплава при тяжелой аварии легководного реактора - внутрикорпусного и внекорпусного - более эффективным является первый, т.е. локализация расплава активной зоны в корпусе реактора (In-Vessel Retention, „IVR“ расплава кориума), при этом основным условием удержания расплава является отсутствие кризиса теплообмена на наружной водоохлаждаемой поверхности корпуса $[1,2,7,8,10,14]$. После формирования на днище корпуса реактора двухжидкостной оксидно-металлической ванны расплава с верхним расположением металлической жидкости (расплав представляет собой систему двух несмешивающихся жидкостей - одна на основе оксидов, другая на основе металлов) наиболее опасным местом с точки зрения возникновения кризиса теплообмена является зона контакта корпуса с металлической жидкостью, в которой фокусируется тепловой поток, передаваемый от оксидной жидкости к металлической $[7,8,10,15,16]$. Величина подводимого от металлического слоя расплава к корпусу реактора теплового потока при прочих равных условиях зависит от сценария тяжелой аварии, уровня остаточного энерговыделения вследствие 
распада присутствующих в расплаве радионуклидов, физико-химических процессов, протекающих в расплаве, а непосредственно - от температуры, теплофизических свойств и толщины металлического слоя расплава.

Одним из возможных физико-химических процессов в высокотемпературном оксидно-металлическом расплаве кориума является его окисление водяным паром или паровоздушной смесью [17-20]. Процесс окисления ванны расплава при верхнем положении металлической жидкости может привести к усилению фокусирующего эффекта из-за дополнительного тепловыделения в металлическом слое за счет экзотермических реакций окисления содержащихся в расплаве восстановителей $(\mathrm{Zr}, \mathrm{U}, \mathrm{Cr}, \mathrm{Fe})$, которое для ВВЭР-1000 может составлять до $50 \%$ от остаточного энерговыделения в расплаве [21]. Учет этого процесса необходим для надежного обоснования удержания расплава в корпусе реактора.

Экспериментальные исследования кинетики окисления расплава кориума были выполнены во ФГУП „НИТИ им. А.П. Александрова“ на экспериментальном комплексе „Расплав“ в рамках проектов АЯЭ/ОЭСР MASCA, MHTЦ METCOR-P, а также CORDEB по контракту IRSN-НИТИ [13,17-19,21-23]. Помимо этого во ФГУП „НИТИ им. А.П. Александрова“ была проведена серия опытов по окислению ванны двухжидкостного субокисленного расплава с верхним положением металлического слоя, в частности, при наличии твердофазного оксидного слоя на поверхности расплава (поверхностной оксидной корки) [20]. Именно такая структура ванны расплава является актуальной на финальной стадии ее формирования. Экспериментально исследованные структуры ванны расплава включали оксидно-металлические системы с верхним положением металлической жидкости, а также металлический расплав, обогащенный $\mathrm{Zr}$ и U. В качестве окислителя использовали смесь $\mathrm{Ar} / \mathrm{O}_{2}$, водяной пар и воздух.

В [20,21] было показано, что большое диффузионное сопротивление корки транспорту окислителя приводит к существенному уменьшению скорости окисления расплава и дополнительного энерговыделения по сравнению с режимом окисления при отсутствии оксидной корки. Тем не менее, поскольку дополнительное энерговыделение за счет экзотермических реакций окисления происходит в металлическом слое, сохраняется необходимость учета их негативного влияния на фокусирующий эффект. Для этого требуется разработка расчетной модели кинетики окисления как составной части расчетных кодов, ориентированных на моделирование тяжелых аварий.

\section{1. Расчетная модель окисления расплава с поверхностной оксидной коркой}

Окисление ванны расплава происходит в реакционной зоне на поверхности металлического расплава. Восстановители $(\mathrm{Zr}, \mathrm{U}, \mathrm{Cr}, \mathrm{Fe})$ перемещаются в реакционной зоне в процессе перераспределения компонентов между нижележащим субокисленным оксидным и поверхностным металлическим слоями ванны расплава, процесс интенсифицируется естественной конвекцией в каждом из сосуществующих слоев. Окислитель из газового объема над расплавом перемещается к расплаву путем диффузии кислорода через твердую оксидную корку. Толщина реакционной зоны определяется балансом между генерацией продуктов окисления и их отводом естественной конвекцией в нижележащий субокисленный оксидный слой ванны расплава [21]. Схема структуры ванны расплава кориума в процессе его окисления приведена на рис. 1.

В экспериментальной ячейке установки „Расплав-3“ геометрические (объем ванны расплава $0.357 \cdot 10^{-3} \mathrm{~m}^{3}$, площадь поверхности ванны $3.848 \cdot 10^{-3} \mathrm{~m}^{2}$, объем газовой фазы над расплавом $5 \cdot 10^{-3} \mathrm{~m}^{3}$, т.е. отношение объема газового пространства к площади поверхности $1.3 \mathrm{~m}^{3} / \mathrm{m}^{2}$ ) и массовые характеристики (масса расплава $2.5 \mathrm{~kg}$ ) радикально отличаются от реакторных условий $[4,24]$, масштаб которых на порядки больше. Однако это не относится к параметрам, определяющим исследуемый процесс окисления.

Для определения коэффициентов диффузии и массопереноса используем первый закон Фика (в пренебрежении температурным градиентом по толщине корки) и закон Аррениуса. Использование законов Фика и Аррениуса в модели окисления оправдано подо-

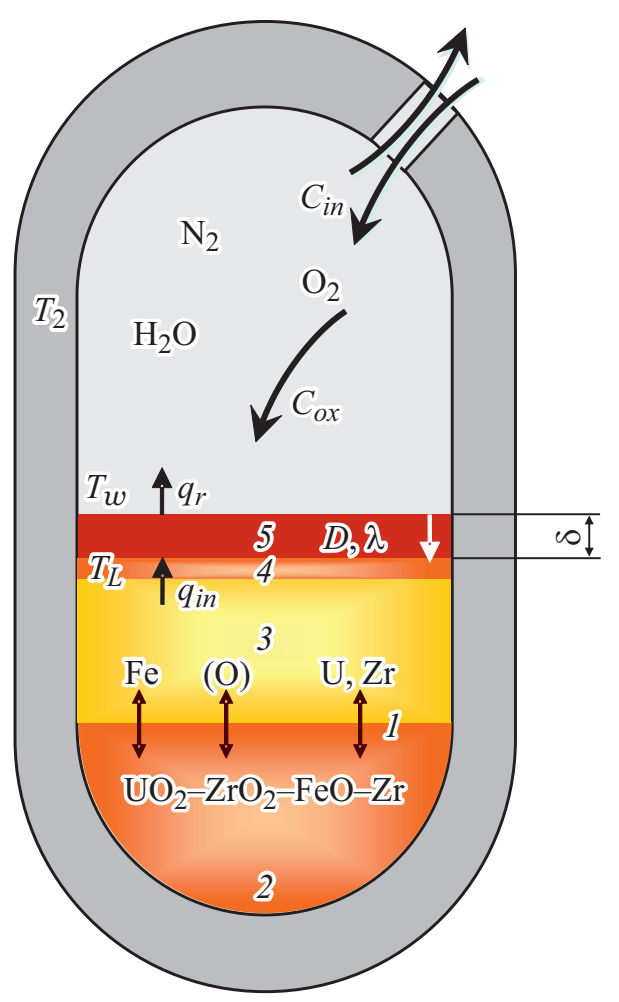

Рис. 1. Схема структуры ванны расплава кориума в корпусе реактора в процессе окисления: 1 - ванна расплава кориума, 2 - оксидный слой, 3 - металлический слой, 4- реакционная зона (жидкие оксиды), 5 - оксидная корка. 
бием условий окисления в экспериментах и в реакторе. Так, для приготовления расплава кориума используются прототипные материалы активной зоны и внутрикорпусных конструкций реактора в заданных пропорциях. Температура расплава и тепловые потоки в оксидную корку также близки к реакторным условиям, а структура ванны расплава им идентична. В качестве окислителя наряду со смесью $\mathrm{Ar} / \mathrm{O}_{2}$ использовались водяной пар и воздух. Поэтому в целом физико-химические процессы окисления расплава кориума на установке „Расплав-3“ и в реакторе можно считать одинаковыми. Значимыми отличиями экспериментальных условий от реакторных могут являться величина газового объема над расплавом и ее отношение к площади поверхности расплава, которое для реакторных условий почти на порядок больше. Однако эти отличия не сказываются непосредственно на процессе окисления, а могут проявиться только в различии распределения компонентов в объеме над расплавом и в инерционности изменения в переходных процессах состава парогазовой атмосферы над расплавом.

Для реакторных условий используемые в дальнейшем анализе параметры $T_{w}, T_{L}$ и $q_{r}$ (температура на наружной поверхности корки, температура ликвидус расплава и тепловой поток, отводимый от наружной поверхности корки соответственно) определяются путем численного моделирования процессов в расплаве кориума, а $C_{o x}$ (концентрация окислителя в объеме над коркой) - путем моделирования термогазодинамических процессов в газовом объеме реактора.

Запишем модель для определения удельной мольной скорости потока окислителя через оксидную корку для условий окисления расплава кориума в установившемся режиме в виде

$$
J_{o x}=-D \frac{\partial C}{\partial x}=D \frac{\Delta C_{o x}}{\delta}
$$

где $J_{o x}$ - удельная мольная скорость переноса окислителя через корку (скорость окисления, так как процесс массопереноса окислителя является лимитирующим для окисления $[18,19]),\left[\mathrm{mol} /\left(\mathrm{m}^{2} \cdot \mathrm{s}\right)\right] ; D-$ коэффициент диффузии окислителя через корку, $\left[\mathrm{m}^{2} / \mathrm{s}\right] ; \delta$ - толщина оксидной корки, $[\mathrm{m}] ; C-$ концентрация окислителя, $\left[\mathrm{mol} / \mathrm{m}^{3}\right] ; \Delta C_{o x}$ - разность концентраций окислителя в объеме над коркой $\left(C_{o x}\right)$ и на границе реакционной зоны, $\left[\mathrm{mol} / \mathrm{m}^{3}\right]$ (рис. 1).

$$
D=A \exp \left(-\frac{E_{a}}{R T_{w}}\right)
$$

где $A$ - предэкспоненциальный множитель (стерический фактор), $\left[\mathrm{m}^{2} / \mathrm{s}\right] ; E_{a}-$ энергия активации, $[\mathrm{J} / \mathrm{mol}]$; $R$ - универсальная газовая постоянная; $T_{w}$ - температура поверхности корки, $[\mathrm{K}]$. Использование именно $T_{w}$ обусловлено тем обстоятельством, что процесс диффузии преимущественно определяется слоем с минимальной температурой, т.е. поверхностным слоем оксидной корки.
Из стационарного уравнения теплопроводности следует, что толщина оксидной корки определяется следующим выражением:

$$
\delta=\frac{\lambda\left(T_{L}-T_{w}\right)}{q_{\text {in }}},
$$

где $\lambda$ - теплопроводность оксидной корки, $[\mathrm{W} /(\mathrm{m} \cdot \mathrm{K})]$; $T_{L}$ - температура ликвидус (монотектики) оксиднометаллического расплава (температура нижней поверхности корки), $[\mathrm{K}] ; q_{\text {in }}$ - кондуктивный тепловой поток через корку, $\left[\mathrm{W} / \mathrm{m}^{2}\right]$.

В установившемся режиме кондуктивный тепловой поток и тепловой поток, отводимый от наружной поверхности корки за счет теплового излучения, равны

$$
q_{\text {in }}=q_{r}=\sigma_{S B}\left[1 / \varepsilon_{w}+1 / \varepsilon_{2}-1\right]^{-1}\left(T_{w}^{4}-T_{2}^{4}\right),
$$

где $\sigma_{S B}-$ постоянная Стефана-Больцмана; $q_{r}-$ лучистый тепловой поток, $\left[\mathrm{W} / \mathrm{m}^{2}\right] ; \varepsilon_{w}-$ излучательная способность поверхности корки, примерно равная 0.8 ; $\varepsilon_{2}$ - излучательная способность поверхностей, воспринимающих тепловое излучение над расплавом, примерно равная единице; $T_{2}$ - температура поверхностей над расплавом, $[\mathrm{K}]$ (для условий проведенных экспериментов усредненное значение $T_{2} \approx 473 \mathrm{~K}$ ).

Для одного из экспериментов (МСР-6) окислителем являлся водяной пар, и выражение (4) приводит к некоторому завышению величины лучистого теплового потока, поскольку в (4) не учитываются поглощение и испускание излучения паром в объеме над расплавом [25]. При определении теплового потока в этом эксперименте полагалось, что влияние указанного фактора невелико вследствие низкой температуры воспринимающих тепловой поток поверхностей, проточной схемы движения пара (отсутствия радиационного равновесия) и небольшой величины (приблизительно $0.5 \mathrm{~m}$ ) толщины парового слоя атмосферного давления.

Подставляя в (1) соотношения (2), (3) и (4), получаем

$$
J_{o x}=A \exp \left(-\frac{E_{a}}{R T_{w}}\right) \frac{q_{r}}{\lambda\left(T_{L}-T_{w}\right)} \Delta C_{o x},
$$

где $q_{r}$ определяется по (4).

Исходя из условий проведенных экспериментов, а именно проточной схемы подачи среды в газовый объем экспериментальной ячейки с многократным превышением расхода окислителя на входе в объем $\left(Q_{i n} C_{i n},[\mathrm{~mol} / \mathrm{s}]\right)$ над его расходом на окисление $\left(G_{o x}\right)$ и определяющей роли поверхностной корки в ограничении скорости окисления, можно считать, что концентрация окислителя над коркой в установившемся режиме приблизительно равна концентрации окислителя на входе:

$$
C_{o x} \approx C_{i n},
$$

где $C_{o x}-$ концентрация окислителя над коркой, $\left[\mathrm{mol} / \mathrm{m}^{3}\right], C_{i n}-$ концентрация окислителя на входе в объем над расплавом, $\left[\mathrm{mol} / \mathrm{m}^{3}\right] ; Q_{i n}-$ суммарный 
Таблица 1. Экспериментальные данные по кинетике окисления расплава кориума при наличии поверхностной оксидной корки

\begin{tabular}{|c|c|c|c|c|c|c|c|}
\hline Тест & Структура ванны & $\begin{array}{c}\text { Температура } \\
\text { поверхности } \\
\text { корки }\left(T_{w}, \mathrm{~K}\right)\end{array}$ & $\begin{array}{c}\text { Температура } \\
\text { ликвидус } \\
\text { расплава }\left(T_{L}, \mathrm{~K}\right)\end{array}$ & Окислитель & $\begin{array}{c}\text { Концентрация } \\
\text { окислителя } \\
\text { на входе } \\
\left(C_{i n}, \mathrm{~mol} / \mathrm{m}^{3}\right)\end{array}$ & $\mid \begin{array}{c}\text { Удельная скорость } \\
\text { окислителя } \\
\text { на входе } \\
\left(Q_{i n} C_{i n} / S \cdot 10^{3}\right. \\
\left.\mathrm{mol} /\left(\mathrm{m}^{2} \cdot \mathrm{s}\right)\right)\end{array}$ & $\begin{array}{c}\text { Удельная скорость } \\
\text { окисления } \\
\left(J_{o x}^{E x p} \cdot 10^{3},\right. \\
\left.\mathrm{mol} /\left(\mathrm{m}^{2} \cdot \mathrm{s}\right)\right)\end{array}$ \\
\hline $\mathrm{OX} 1(3 / 1)$ & \multirow{7}{*}{$\begin{array}{c}\text { Оксидно- } \\
\text { металлическая } \\
\text { ванна расплава } \\
\text { с верхним } \\
\text { расположением } \\
\text { металлического } \\
\quad \text { слоя }\end{array}$} & 2075 & \multirow{4}{*}{2625} & \multirow{6}{*}{$\begin{array}{l}\mathrm{Ar} / \mathrm{O}_{2} \\
\text { смесь }\end{array}$} & $0.9 \pm 0.1$ & 40.0 & $10.2 \pm 1.5$ \\
\hline $\mathrm{OX} 1(3 / 2)$ & & 2000 & & & $8.6 \pm 0.1$ & 385.0 & $63.7 \pm 9.6$ \\
\hline $\mathrm{OX} 1(3 / 3)$ & & 2010 & & & $12.9 \pm 0.1$ & 583.0 & $96.6 \pm 14.5$ \\
\hline $\mathrm{OX} 1(3 / 4)$ & & & & & $17.2 \pm 0.1$ & 760.0 & $143.7 \pm 21.6$ \\
\hline CD2-01(2) & & \multirow{2}{*}{2075} & 2775 & & \multirow{2}{*}{$8.3 \pm 0.1$} & \multirow{2}{*}{375.0} & $9.1 \pm 6.5$ \\
\hline $\mathrm{CD} 2-02$ & & & 2625 & & & & $86 \pm 13$ \\
\hline MA-7(2) & & 2315 & 3025 & воздух & $41.7 \pm 1.0$ & 1770.0 & $360 \pm 70$ \\
\hline MCP-6 & $\begin{array}{c}\text { Расплав стали, } \\
\text { обогащенный } \\
\mathrm{U} \text { и } \mathrm{Zr}\end{array}$ & 1450 & 1575 & пар & $30.0 \pm 1.0$ & 300.0 & $67 \pm 7$ \\
\hline
\end{tabular}

объемный расход окислителя и сопутствующего газа $\left(\mathrm{Ar} / \mathrm{O}_{2}\right.$, пар, воздух $),\left[\mathrm{m}^{3} / \mathrm{s}\right] ; G_{o x}-$ расход окислителя, затрачиваемого на окисление расплава, $[\mathrm{mol} / \mathrm{s}]$.

Вследствие того что при окислении расплава кориума через оксидную корку поступающий в реакционную зону окислитель полностью взаимодействует с восстановителем, причем скорость окисления (расход идущего на взаимодействие с расплавом окислителя) не изменяется по мере уменьшения концентрации восстановителей в расплаве $[18,19]$, концентрация окислителя в реакционной зоне (на интерфейсе корка-расплав) близка к нулевой величине. Тогда перепад концентрации окислителя между газовым объемом (над коркой) и реакционной зоной

$$
\Delta C_{o x} \approx C_{i n},
$$

и зависимость (5) для условий экспериментов принимает вид

$$
J_{o x}=A \exp \left(-\frac{E_{a}}{R T_{w}}\right) \frac{q_{r}}{\lambda\left(T_{L}-T_{w}\right)} C_{i n},
$$

где $J_{o x}=G_{o x} / S$ - удельная мольная скорость окисления, $\left[\mathrm{mol} /\left(\mathrm{m}^{2} \cdot \mathrm{s}\right)\right] ; S-$ площадь поверхности оксидной корки, $\left[\mathrm{m}^{2}\right]$.

\section{2. Определение параметров модели и обсуждение результатов}

Для удобства дальнейшего анализа условия проведения опытов и экспериментальные результаты по скорости окисления расплава кориума при наличии оксидной корки $[18,19,21]$ систематизированы в табл. 1.

Решение обратной задачи нахождения неизвестных коэффициента и параметра в выражении (8) выполнялось по экспериментальным данным, приведенным в табл. 1, и позволило определить коэффициент $A\left(0.9 \cdot 10^{-3} \mathrm{~m}^{2} / \mathrm{s}\right)$ и величину энергии активации процесса окисления $E_{a}$ $(70.3 \mathrm{~kJ} / \mathrm{mol})$. Следует отметить, что полученное значение энергии активации близко к энергии активации самодиффузии кислорода в стабилизированной кубической модификации $\mathrm{ZrO}_{2}(111 \pm 20$ [26], $70 \pm 12 \mathrm{~kJ} / \mathrm{mol}$ [27]). Энергия активации самодиффузии кислорода в $\mathrm{UO}_{2}$ несколько выше ( 145 [28], $169 \pm 75 \mathrm{~kJ} / \mathrm{mol}$ [29]). Таким образом, можно предполагать, что в твердом растворе на основе $(\mathrm{U}, \mathrm{Zr}) \mathrm{O}_{2}$ преобладает механизм, свойственный суперионным проводникам по кислороду, таким как стабилизированный диоксид циркония. Среднее значение (для средней в проведенных опытах величины $T_{w}$ ) коэффициента диффузии, полученное исходя из выполненных оценок величин энергии активации и предэкспоненциального множителя, составило $1.38 \cdot 10^{-4} \mathrm{~cm}^{2} / \mathrm{s}$, что более чем на два порядка отличается (в большую сторону) как от имеющихся экспериментальных данных по самодиффузии ионов кислорода по твердой фазе $\mathrm{UO}_{2}$, обобщение которых приведено в работе [29], так и по самодиффузии ионов кислорода по твердой фазе кубического твердого раствора на основе $\mathrm{ZrO}_{2}$ [30].

Причиной этого может быть трещинообразование в корке, которое обусловлено увеличением объема расплава при окислении кислородом, поступающим через оксидную корку путем диффузии ионов, а также наличием других несплошностей образующейся оксидной корки, что может кардинальным образом сказаться на стерическом факторе, оставляя значение энергии активации диффузии кислорода тем же, что и при диффузии ионов через сплошную твердую фазу.

Через возникающие в оксидной корке трещины на поверхность корки из реакционной зоны выдавливается часть расплава с температурой выше $T_{L}$, кото- 
Таблица 2. Расчетные и экспериментальные значения удельной скорости окисления

\begin{tabular}{c|c|c|c|c|c|c|c|c}
\hline \multirow{2}{*}{$J_{\text {ox }} \cdot 10^{3}, \mathrm{~mol} /\left(\mathrm{m}^{2} \cdot \mathrm{s}\right)$} & \multicolumn{7}{c}{ Tec } \\
\cline { 2 - 9 } & OX1(3/1) & OX1(3/2) & OX1(3/3) & OX1(3/4) & CD2-01(2) & CD2-02 & MA-7(2) & MCP-6 \\
\hline$J_{\text {Calc }}^{\text {Cax }}$ & $11.3 \pm 2.4$ & $66.0 \pm 13.7$ & $104.5 \pm 21.7$ & $139.0 \pm 28.8$ & $75.9 \pm 16.1$ & $97.8 \pm 20.8$ & $364.5 \pm 110.4$ & $65.2 \pm 10.5$ \\
$J_{o x}^{E x p}$ & $10.2 \pm 1.5$ & $63.7 \pm 9.6$ & $96.6 \pm 14.5$ & $143.7 \pm 21.6$ & $9.1 \pm 6.5$ & $86 \pm 13$ & $360 \pm 70$ & $67 \pm 7$
\end{tabular}

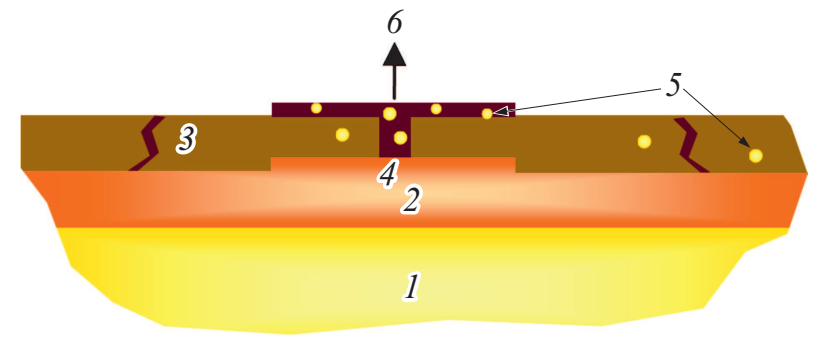

Pис. 2. Схема движения оксидной корки за счет кристаллизации расплава, поступающего через трещины в корке, на ее поверхности и плавления нижней поверхности корки: $1-$ металлический слой ванны расплава кориума, 2 - реакционная зона (жидкие оксиды), 3 - оксидная корка, 4 - плавление оксидной корки, 5 - металлические включения, 6 - перемещение оксидной корки.

рая окисляется и кристаллизуется с высокой скоростью, существенно увеличивая скорость взаимодействия расплава с кислородом и сильно влияя на величину предэкспоненциального множителя. При этом толщина оксидной корки остается постоянной, так как увеличение толщины корки за счет кристаллизации выдавленного на поверхность расплава при постоянном тепловом потоке, подводимом от расплава к оксидной корке, компенсируется плавлением нижнего слоя корки (рис. 2).

По результатам анализа микроструктуры, выполненного обработкой микрофотографий, полученных методом сканирующей электронной микроскопии, пористость оксидной корки в проведенных опытах (кроме МА-7(2)) изменялась в пределах 5-10\% при среднем размере пор от 6 до $70 \mu \mathrm{m}$. На основании экспериментальных данных $[13,15,31,32]$ величина теплопроводности принималась равной $2 \mathrm{~W} /(\mathrm{m} \cdot \mathrm{K})$ с оцененной неопределенностью $\pm 15 \%$.

Отметим, что использование для определения теплопроводности корки экспериментальных данных, полученных в близких для опытов по окислению расплава условиях по температуре, материалу, пористости и размеру пор корки, позволяет отказаться от расчетного определения эффективной теплопроводности, учитывающего распространение теплового излучения в пористой полупрозрачной среде [33-36].

При окислении компонентов расплава воздухом (опыт МА-7(2)) образовавшаяся корка имеет малую пористость $(0.6 \%)$ со средним размером пор $6 \mu \mathrm{m}$. Вследствие взаимодействия восстановителей расплава и с кислородом, и с азотом в корке присутствуют фазы не только на основе оксидов, но и оксинитридные и нитридные фазы урана и циркония, которые имеют теплопроводность в диапазоне $8-30 \mathrm{~W} /(\mathrm{m} \cdot \mathrm{K})$, а сам расплав имеет высокую температуру ликвидус $\left(T_{L}\right)$. Значение $\lambda$ корки в опыте МА-7(2) в приближении аддитивности по отношению к содержащимся в корке компонентам, в предположении преобладания оксинитридных фаз, составляет $5 \mathrm{~W} /(\mathrm{m} \cdot \mathrm{K})$ с оцененной неопределенностью $\pm 25 \%$.

Окончательно выражение для скорости окисления имеет вид

$$
J_{o x}=B \exp \left[-\frac{E_{a}}{R T_{w}}\right] \frac{\left(T_{w}^{4}-T_{2}^{4}\right)}{\lambda\left(T_{L}-T_{w}\right)} C_{i n},
$$

где $B=A \sigma_{S B}\left[1 / \varepsilon_{w}+1 / \varepsilon_{2}-1\right]^{-1}=4.09 \cdot 10^{-11} \mathrm{~W} /\left(\mathrm{s} \cdot \mathrm{K}^{4}\right)$ при $\varepsilon_{w}=0.8, \varepsilon_{2}=1$.

Сравнение расчетных значений удельной скорости окисления $\left(J_{o x}^{\text {Calc }}\right)$ с экспериментальными значениями $\left(J_{o x}^{E x p}\right)$ приведено в табл. 2 и на рис. 3.

Как видно из табл. 2 и рис. 3, выражение (9) с высокой точностью описывает имеющийся массив экспериментальных данных по скорости окисления расплава

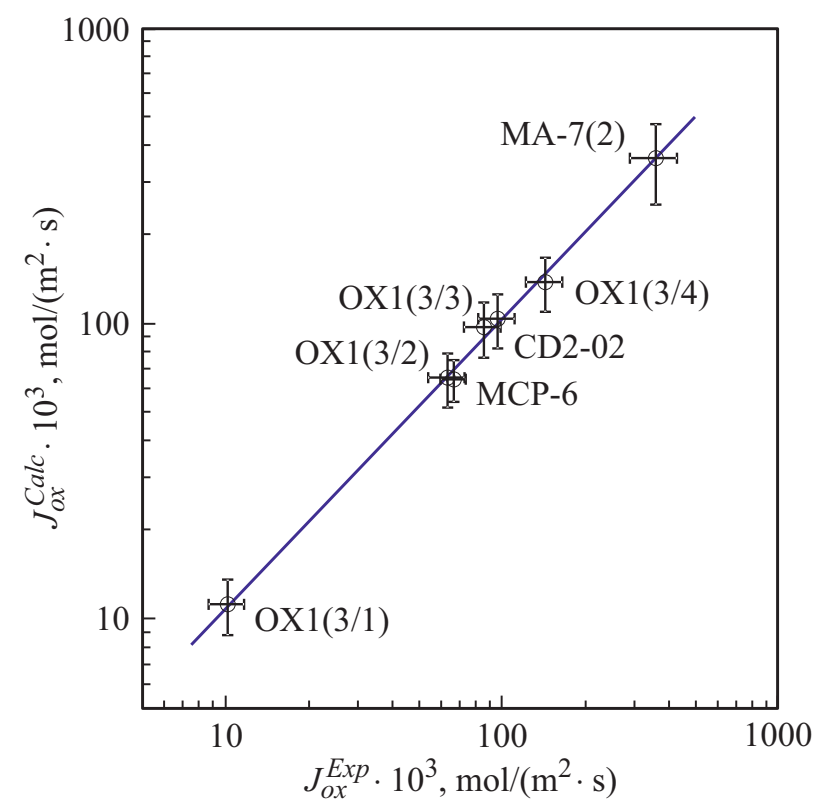

Рис. 3. Сопоставление расчетных $\left(J_{o x}^{\text {Calc }}\right)$ и экспериментальных $\left(J_{o x}^{E x p}\right)$ значений удельной скорости окисления расплава через оксидную корку. 


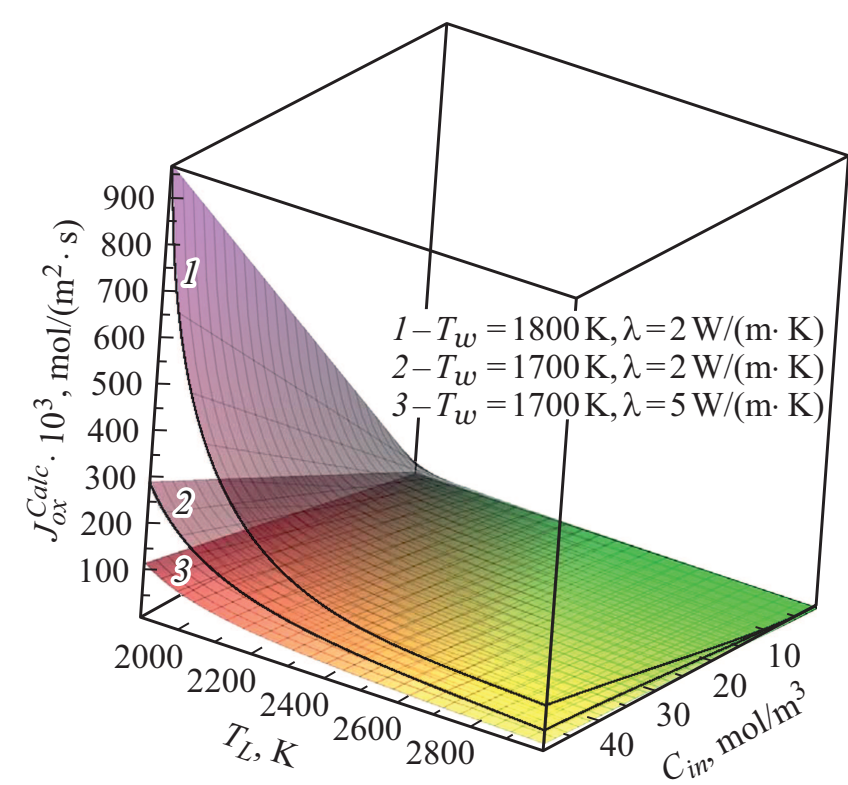

Рис. 4. Расчетные зависимости скорости окисления $\left(J_{o x}^{C a l c}\right)$ от температуры ликвидус расплава $\left(T_{L}\right)$ и концентрации окислителя на входе $\left(C_{i n}\right)$ при различной температуре поверхности $\left(T_{w}\right)$ и теплопроводности оксидной корки $(\lambda)$.

кориума с поверхностной коркой. Анализ расчетных оценок скорости окисления показывает, что наиболее чувствительным параметром выражения (9) является теплопроводность оксидной корки, неопределенность значения которой не превышает 25\%. Приведенные в [37] результаты бенчмарка IVR-кодов свидетельствуют, что разброс значений исходных параметров моделей может превышать 50\%. Это может приводить к вариации расчетных оценок до 70\% от средней величины. Таким образом, предложенная расчетная зависимость может быть интегрирована в существующие расчетные коды без существенного увеличения неопределенности расчетных оценок [37].

При обобщении опытных данных не использовались результаты опыта CD2-01(2), в котором в отличие от всех других опытов корка формировалась захолаживанием поверхностного слоя расплава не в окислительной, а в нейтральной атмосфере, и образование твердых оксидов происходило за счет содержащегося в металлическом расплаве кислорода. Указанное отличие привело к изменению процесса трещинообразования, которое началось позже, чем в других опытах (при большей толщине корки), и при подаче окислителя количество трещин оказалось существенно меньшим. Этим объясняется существенно меньшая скорость окисления расплава в опыте CD2-01(2).

На рис. 4 приведены полученные с использованием выражения (9) примеры расчетной зависимости удельной скорости окисления от температуры поверхности и теплопроводности оксидной корки, температуры ликвидус расплава и концентрации окислителя. Можно видеть, что скорость окисления резко увеличивается при повышении концентрации окислителя в газовой фазе и температуры поверхности корки, понижении теплопроводности и температуры ликвидус расплава.

Применение полученной зависимости для реакторных условий требует отказа от использованного для условий проведенных экспериментов равенства (6) и определения концентрации окислителя над коркой $C_{o x}$ путем моделирования термогазодинамических процессов в газовом объеме реактора. Соответственно в правой части выражения (9) вместо $C_{\text {in }}$ используется $C_{o x}$. При этом среднее (среднеинтегральное) значение концентрации окислителя в газовом объеме реактора $C_{0}$ в общем случае контролируется решением уравнения

$$
V \frac{d C_{0}}{d \tau}=Q_{i n} C_{i n}-J_{o x} S
$$

где $V$ - объем газового пространства, $\left[\mathrm{m}^{3}\right]$.

При $Q_{i n} C_{i n} \neq 0$ концентрация окислителя в объеме газовой фазы над расплавом изменяется во времени, пока скорость окисления не выйдет на стационарное значение, соответствующее расходу окислителя в объеме над расплавом. При $Q_{i n} C_{i n}=0$ скорость окисления снижается до нулевого значения при полном израсходовании окислителя в объеме газовой фазы.

Следует отметить необходимость дальнейшего уточнения теплофизических, термодинамических и структурных характеристик корки, что приведет к корректировке значений параметров $\lambda, B$ и $E_{a}$ в выражении (9) и позволит сделать прогнозы расчетных кодов более точными.

\section{Заключение}

Предложенная на основе модели диффузии с использованием экспериментальных данных зависимость для расчета окисления расплава активной зоны ядерного реактора в присутствии на его поверхности оксидной корки имеет простую структуру и оптимальна для применения в расчетных кодах, моделирующих тяжелые аварии. Параметры, входящие в выражение (9), определяются из решения термогазодинамических и термодинамических уравнений в указанных кодах.

Учет процесса окисления в расчетных кодах позволит повысить точность расчетной оценки фокусирующего эффекта и, следовательно, надежность обоснования удержания расплава кориума в охлаждаемом корпусе реактора.

Применимость полученных в маломасштабных экспериментах результатов для натурных условий тяжелой аварии обусловлена использованием в экспериментах химически прототипного состава кориума, а также незначительным влиянием массообмена в газовом (паровом) объеме на скорость окисления при наличии поверхностной корки. Различие трещинообразования может в соответствии с масштабным фактором проявиться в уменьшении числа, ширины и протяженности трещин, приходящихся на единицу площади корки, т.е. в 
уменьшении вклада трещин в массоперенос окислителя через корку и соответственно в уменьшении скорости окисления. В этом случае полученная зависимость даст верхнюю, консервативную оценку.

Зависимость прогнозных значений скорости окисления расплава кориума от состава газовой фазы свидетельствует о целесообразности расширения базы экспериментальных данных по взаимодействию расплава с газовыми средами различного состава, в частности, по окислению расплава водяным паром, в том числе при пленочном кипении воды на поверхности корки. Некоторое влияние на погрешность прогноза оказывает и неопределенность в данных по теплопроводности оксидной корки. Однако следует отметить, что предложенная в работе зависимость может быть адаптирована к новым данным без существенных изменений.

\section{Финансирование работы}

Экспериментальная работа, на основе которой построена математическая модель окисления, выполнена при частичной финансовой поддержке МНТЦ, АЯЭ/ОЭСР и IRSN.

\section{Конфликт интересов}

Авторы заявляют, что у них нет конфликта интересов.

\section{Список литературы}

[1] Nuclear Safety in Light Water Reactors: Severe Accident Phenomenology. Ed. by B.R. Sehgal. (Academic Press, Elsevier, 2012). DOI: 10.1016/C2010-0-67817-5

[2] Nuclear power reactor core melt accidents. Current state of knowledge. Ed. by D. Jacquemain. (EDP Sciences, France, 2015).

[3] V.G. Asmolov, I.N. Gusev, V.R. Kazanskiy, V.P. Povarov, D.B. Statsura. Nucl. Energy and Technol., 3 (4), 260 (2017). DOI: 10.1016/j.nucet.2017.10.003

[4] В.В. Гусаров, В.И. Альмяшев, В.Б. Хабенский, С.В. Бешта, В.С. Грановский. Рос. хим. ж., XLIX (4), 42 (2005).

[5] A.A. Komlev, V.I. Almjashev, S.V. Bechta, V.B. Khabensky, V.S. Granovsky, V.V. Gusarov. J. Nucl. Mater., 467, 778 (2015). DOI: 10.1016/j.jnucmat.2015.10.035.

[6] Основные принципы безопасности атомных электростанщий 75-INSAG-3 Rev. 1. Доклад Межсдународной консультативной группы по ядерной безопасности. INSAG-12. STI/PUB/1082. (МАГАТЭ, Вена, 2015).

[7] F. Fichot, L. Carénini, M. Sangiorgi, S. Hermsmeyer, A. Miassoedov, S. Bechta, J. Zdarek, D. Guenadou. Annals of Nucl. Energy, 119, 36 (2018). DOI: 10.1016/j.anucene.2018.03.040

[8] V.I. Almjashev, V.S. Granovsky, V.B. Khabensky, S.Yu. Kotova, E.V. Krushinov, A.A. Sulatsky, S.A. Vitol, V.V. Gusarov, F. Fichot, B. Michel, P. Piluso, R. Le Tellier, M. Fischer, C. Le Guennic, N. Bakouta. Nucl. Eng. and Des., 332, 31 (2018). DOI: 10.1016/j.nucengdes.2018.03.004
[9] V.B. Khabensky, V.S. Granovsky, V.I. Almjashev, S.A. Vitol, E.V. Krushinov, S.Ju. Kotova, A.A. Sulatsky, V.V. Gusarov, S.V. Bechta, M. Barrachin, D. Bottomley, M. Fischer, S. Hellmann, P. Piluso, A. Miassoedov, W. Tromm. Nucl. Eng. and Des., 327, 82 (2018). DOI: $10.1016 /$ j.nucengdes.2017.11.030

[10] L. Carénini, F. Fichot, N. Seignour. Annals of Nucl. Energy, 118, 363 (2018). DOI: 10.1016/j.anucene.2018.04.032

[11] K. Plevacova, C. Journeau, P. Piluso, V. Zhdanov, V. Baklanov, J. Poirier. J. Nucl. Mater., 414 (1), 23 (2011). DOI: $10.1016 /$ j.jnucmat.2011.04.055

[12] A. Quaini, S. Gossé, C. Guéneau, L. Soldi, D. Manara. J. Nucl. Mater., 509, 517 (2018). DOI: 10.1016/j.jnucmat.2018.07.021

[13] РАСПЛАВ. Удержсание расплавленных материалов активной зоны водоохлаждаемых реакторов (международные проекты RASPLAV и MASCA). Под ред. В.Г. Асмолова, А.Ю. Румянцева, В.Ф. Стрижова. (Концерн Росэнергоатом, М., 2018).

[14] S.V. Bechta, V.S. Granovsky, V.B. Khabensky, E.V. Krushinov, S.A. Vitol, V.F. Strizhov, D. Bottomley, M. Fischer, P. Piluso, A. Miassoedov, W. Tromm, E. Altstadt, H.G. Willschutz, F. Fichot, O. Kymalainen. Proceedings of the $3^{\text {rd }}$ Europian Review Meeting on Severe Accident Research (ERMSAR2008) (Nesseber, Bulgaria, 23-25 September, 2008). Corium Session 2 - Paper 2.7.

[15] T.G. Theofanous, C. Liu, S. Additon, S. Angelini, O. Kymäläinen, T. Salmassi. Nucl. Eng. Des., 169 (1-3), 1 (1997). DOI: 10.1016/S0029-5493(97)00009-5

[16] Л.А. Домбровский, Л.И. Зайчик, Ю.А. Зейгарник. Теплоэнергетика, 45 (9), 43 (1998). eLIBRARY ID: 27017298. [L.A. Dombrovskii, L.I. Zaichik, Yu.A. Zeigarnik. Therm. Eng., 45 (9), 755 (1998). eLIBRARY ID: 13296953].

[17] A.A. Sulatsky, S.A. Smirnov, V.S. Granovsky, V.B. Khabensky, E.V. Krushinov, S.A. Vitol, S.Yu. Kotova, M. Fischer, S. Hellmann, W. Tromm, A. Miassoedov, D. Bottomley, P. Piluso, M. Barrachin. Nucl. Eng. Des., 262, 168 (2013). DOI: 10.1016/j.nucengdes. 2013.04.025

[18] V.S. Granovsky, V.B. Khabensky, E.V. Krushinov, S.A. Vitol, A.A. Sulatsky, V.I. Almjashev, S.V. Bechta, V.V. Gusarov, M. Barrachin, P.D. Bottomley, M. Fischer, P. Piluso. Nucl. Eng. Des., 278, 310 (2014). DOI: 10.1016/j.nucengdes.2014.07.034

[19] V.I. Almjashev, V.S. Granovsky, V.B. Khabensky, E.V. Krushinov, A.A. Sulatsky, S.A. Vitol, V.V. Gusarov, S. Bechta, M. Barrachin, F. Fichot, P.D. Bottomley, M. Fischer, P. Piluso. Nucl. Eng. Des., 305, 389 (2016). DOI: 10.1016/j.nucengdes.2016.05.024

[20] А.А. Сулацкий, В.Б. Хабенский, В.С. Грановский, В.И. Альмяшев, Е.В. Крушинов, Е.В. Шевченко, С.А. Витоль, С.Ю. Котова, Е.К. Каляго, Е.Б. Шуваева, В.Р. Булыгин, Е.М. Беляева. Технол. обесп. жизн. цикла ядерн. энергет. устан., 1 (15), 66 (2019). eLIBRARY ID: 37643366.

[21] A.A. Sulatsky, V.I. Almjashev, V.S. Granovsky, V.B. Khabensky, E.V. Krushinov, S.A. Vitol, V.V. Gusarov, F. Fichot, B. Michel, P. Piluso, R. Le Tellier, C. Le Guennic, N. Bakouta. Nucl. Eng. Des., 363, 110618 (2020). DOI: 10.1016/j.nucengdes.2020.110618

[22] S.V. Bechta, V.B. Khabensky, S.A. Vitol, E.V. Krushinov, V.S. Granovsky, D.B. Lopukh, V.V. Gusarov, A.P. Martinov, V.V. Martinov, G. Fieg, W. Tromm, D. Bottomley, H. Tuomisto. Nucl. Eng. Des., 236, 1362 (2006). DOI: $10.1016 /$ j.nucengdes.2005.12.008 
[23] S.V. Bechta, E.V. Krushinov, S.A. Vitol, V.B. Khabensky, S.Yu. Kotova, A.A. Sulatsky, V.V. Gusarov, V.I. Almyashev, G. Ducros, C. Journeau, D. Bottomley, B. Clément, L. Herranz, S. Guentay, K. Trambauer, A. Auvinen, V.V. Bezlepkin. Nucl. Eng. Des., 240, 1229 (2010). DOI: $10.1016 /$ j.nucengdes.2010.01.008

[24] С.А. Андрушечко, А.М. Афров, Б.Ю. Васильев, В.Н. Генералов, К.Б. Косоуров, Ю.М. Семченков, В.Ф. Украинцев. АЭС с реактором типа ВВЭР-1000. От физических основ эксплуатащии до эволющии проекта. (Логос, М., 2010).

[25] Л.А. Домбровский. Теплоэнергетика, 48 (1), 42 (2001). eLIBRARY ID: 27155864. [L.A. Dombrovskii. Therm. Eng., 48 (1), 42 (2001). eLIBRARY ID: 13383037].

[26] В.Г. Заводинский. ФТТ. 46 (3), 441 (2004). [V.G. Zavodinsky. Phys. Solid State., 46, 453 (2004) DOI: 10.1134/1.1687859]

[27] C. Yang, K. Trachenko, S. Hull, I.T. Todorov, M.T. Dove. Physical Review B. 97 (18), 184107 (2018). DOI: 10.1103/PhysRevB.97.184107

[28] А.Ю. Куксин, Д.Е. Смирнова. ФТТ. 56 (6), 1166 (2014). [A.Yu. Kuksin, D.E. Smirnova. Phys. Solid State., 56, 1214 (2014) DOI: 10.1134/S1063783414060201]

[29] S.I. Potashnikov, A.S. Boyarchenkov, K.A. Nekrasov, A.Ya. Kupryazhkin. J. Nucl. Mater., 433 (1-3), 215 (2013). DOI: 10.1016/j.jnucmat.2012.08.033

[30] V.V. Sizov, M.J. Lampinen, A. Laaksonen. Solid State Ion., 266, 29 (2014). DOI: 10.1016/j.ssi.2014.08.003

[31] V.F. Strizhov, R.G. Galimov, V.D. Ozrin, V.Y. Zitserman, G.A. Kobzev, L.R. Fokin, P. Piluso, H. Chalaye. Societe Francaise d'Energie Nucleaire - International Congress on Advances in Nuclear Power Plants - ICAPP 2007, The Nuclear Renaissance at Work. (Nice, France, 2007). P. 2692.

[32] A. Seibert, D. Staicu, D. Bottomley, M. Cologna, J. Boshoven, H. Hein, E. Kassim, S. Nourry, M. Ernstberger, D. Robba, R. Konings. J. Nucl. Mater., 520, 165 (2019). DOI: 10.1016/j.jnucmat.2019.04.019

[33] L.A. Dombrovsky, B. Rousseau, P. Echegut, J.H. Randrianalisoa, D. Baillis. J. Amer. Ceramic Soc. 94 (12), 4310 (2011). DOI: $10.1111 / \mathrm{j} .1551-2916.2011 .04655 . x$

[34] M. Colbert, F. Ribeiro, G. Tréglia. J. Appl. Phys. 115, 034902 (2014). DOI: $10.1063 / 1.4861418$

[35] L. He, M. Zhang, H. Gu, A. Huang. Interceram. 65 (6-7), 238 (2018). DOI: 10.1007/BF03401175

[36] K.B. Kiradjiev, S.A. Halvorsen, R.A. Van Gorder, S.D. Howison. Int. J. Therm. Sci. 145, 106009 (2019). DOI: 10.1016/j.ijthermalsci.2019.106009

[37] L. Carénini, F. Fichot, N. Bakouta, A. Filippov, R. Le Tellier, L. Viot, I. Melnikov, P. Pandazis. Ann. Nucl. Energy. 146, 107612 (2020). DOI: 10.1016/j.anucene.2020.107612 